\title{
Polishing Sapphire Substrates by 355 nm Ultraviolet Laser
}

\author{
X. Wei, X. Z. Xie, W. Hu, and J. F. Huang \\ School of Electromechanical Engineering, Guangdong University of Technology, Guangzhou 510090, China \\ Correspondence should be addressed to X. Wei, weixin@gdut.edu.cn
}

Received 15 February 2012; Revised 21 May 2012; Accepted 29 May 2012

Academic Editor: Jian Xu

Copyright ( $12012 \mathrm{X}$. Wei et al. This is an open access article distributed under the Creative Commons Attribution License, which permits unrestricted use, distribution, and reproduction in any medium, provided the original work is properly cited.

This paper tries to investigate a novel polishing technology with high efficiency and nice surface quality for sapphire crystal that has high hardness, wear resistance, and chemical stability. A Q-switched $355 \mathrm{~nm}$ ultraviolet laser with nanosecond pulses was set up and used to polish sapphire substrate in different conditions in this paper. Surface roughness $R_{a}$ of polished sapphire was measured with surface profiler, and the surface topography was observed with scanning electronic microscope. The effects of processing parameters as laser energy, pulse repetition rate, scanning speed, incident angle, scanning patterns, and initial surface conditions on surface roughness were analyzed.

\section{Introduction}

Sapphire is a kind of important photoelectronic material with excellent properties such as high hardness, wear resistance, and chemical stability. So it is applied not only as important substrate for GaN and then LED, but also as important components for solid lasers, IR window and precision bearing. All these applications require sapphire surface with high machining quality and thin damaged layer [1]. Conventional polishing techniques as abrasive polishing, chemical mechanical polishing (CMP), and chemical polishing [2] are very difficult to get high quality for sapphire crystal polishing due to machining defects like mechanical scratch, low efficiency, and yield. Laser polishing technology shows potential for sapphire crystal because of high polishing quality and efficiency. Recently, series of studies on laser polishing of diamond film, polymer, ceramics, fiber, and metal have been conducted [3-7]. However, the research on laser polishing of sapphire crystal is still lack.

Polishing sapphire substrate [8] by ultraviolet laser with picosecond pulses had been conducted in our previous research. The results indicated that better surface quality can be achieved via shorter wavelength and pulse duration. However, the high cost of picosecond ultraviolet lasers affects its further industrialization. Currently, the Q-switched ultraviolet laser with wavelength of $355 \mathrm{~nm}$ and nanosecond pulses is widely introduced to industry. Such type of ultraviolet lasers can be used to machine and remove sapphire material in micrometer scale. Thus, a Q-switched $355 \mathrm{~nm}$ ultraviolet laser with nanosecond pulses was used to polish sapphire substrate in this paper. The effects of processing parameters as laser energy, pulse repetition rate, laser scanning speed, incident angle, scanning patterns, and initial sapphire surface conditions on surface quality were analysed according to the measurement of surface roughness $R_{a}$ by surface profiler and the observation of surface topography by scanning electronic microscope. The optimized processing parameters were obtained by orthogonal experiments.

\section{Experimental Setup}

Figure 1 shows schematically the laser polishing system. The experimental system mainly included an ultraviolet laser source, optical system, the work table, and the PC-based controller. Movements of the work table were controlled by a computer. Laser beam was delivered by the optical system and focused by f-theta focusing lens on the specimen surface. Hence, the focusing beam could ablate the surface of sapphire sample. The sapphire sample was fixed on the work table and the angle between the sapphire surface and the light beam was adjusted by inclining the work table.

The laser polishing system consisted of a ultraviolet laser operator with $355 \mathrm{~nm}$ wavelength and laser mode of TEM00, average power of 2 Watts, pulse width of $20 \mathrm{~ns}$, pulse 


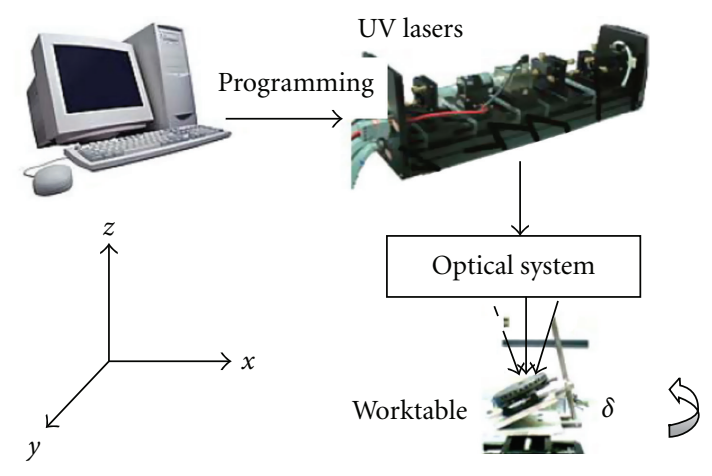

FIgURE 1: Schematic diagram of laser polishing system.

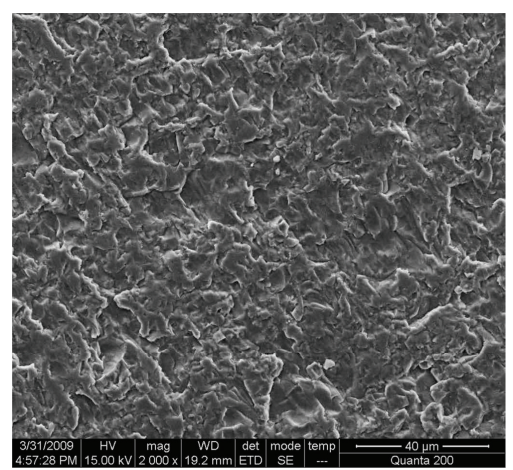

Sample A

(a)

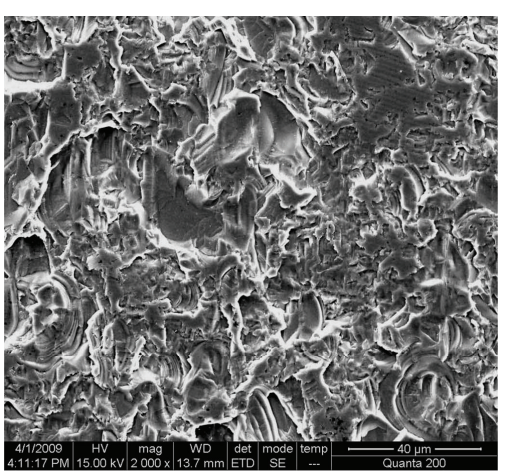

Sample B

(b)

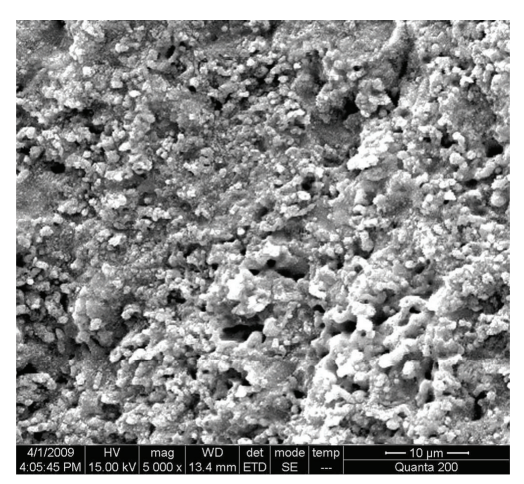

Sample C

(c)

FIGURE 2: Samples used in this research.

TABLE 1: The laser parameters.

\begin{tabular}{ll}
\hline Laser energy $(\mathrm{mJ})$ & $0.03,0.036,0.044,0.050,0.056$, \\
& $0.06,0.064$ \\
Laser pulse repetition rate $(\mathrm{kHz})$ & $10,20,30,40,50,60$ \\
Laser scanning speed $(\mathrm{mm} / \mathrm{s})$ & $10,30,50,70,90,110,130$ \\
Incident angle $\left(^{\circ}\right)$ & $0,10,30,50$ \\
Scanning patterns & A, B, C, D, E \\
\hline
\end{tabular}

repetition rate of $100 \mathrm{kHz}$, scanning speed of $700 \mathrm{~mm} / \mathrm{s}$, and focal length of $14 \mu \mathrm{m}$.

Three sapphire samples (see Figure 2) were prepared as follows.

(1) Sample A: mechanical lapped surface, with dimension of $60 \times 40 \times 2 \mathrm{~mm}$ and surface roughness $R_{a}$ $0.75 \mu \mathrm{m}$.

(2) Sample B: sawed surface, with dimension of $18 \times 10$ $\times 1.5 \mathrm{~mm}$ and surface roughness $R_{a} 1.1 \mu \mathrm{m}$.

(3) Sample C: processed surface by $532 \mathrm{~nm}$ green laser, with dimension of $60 \times 40 \times 2 \mathrm{~mm}$ and surface roughness $R_{a} 1.5 \mu \mathrm{m}$.

The processing parameters were selected as listed in Table 1 .
For evaluating the polishing results, the surface roughness $R_{a}$ of the polished sapphire substrate was measured by a surface profiler (MarSurf XR20) and the surface topography was observed by a scanning electronic microscope.

\section{Results and Discussions}

3.1. Effects of Laser Energy on Surface Roughness. Figure 3(a) shows the effect of laser energy on surface roughness of the polished sapphire. With the increase of laser energy, the surface roughness $R_{a}$ decreases when laser energy is lower than $0.056 \mathrm{~mJ}$ and increases when the energy is higher than $0.056 \mathrm{~mJ}$.

When the laser with pulse energy of $0.064 \mathrm{~mJ}$ was used, the polished sapphire surface consisted mainly of microcracks caused by thermal stress because of the rapid resolidification, as shown in Figure 3(b). If a small laser energy like $0.044 \mathrm{~mJ}$ was applied, the polished sapphire surface was observed with the features of submicropores or ablated pits, and micromelting and resolidification structure. The ablated pits were caused mainly by photoionization and impact ionization. However, there were few sign of microcrack in this energy condition, as shown in Figure 3(c). These results showed that the sapphire material radiated by small pulse energy laser is mainly removed by thermal and photochemical action together, and if radiated by high pulse energy laser, 


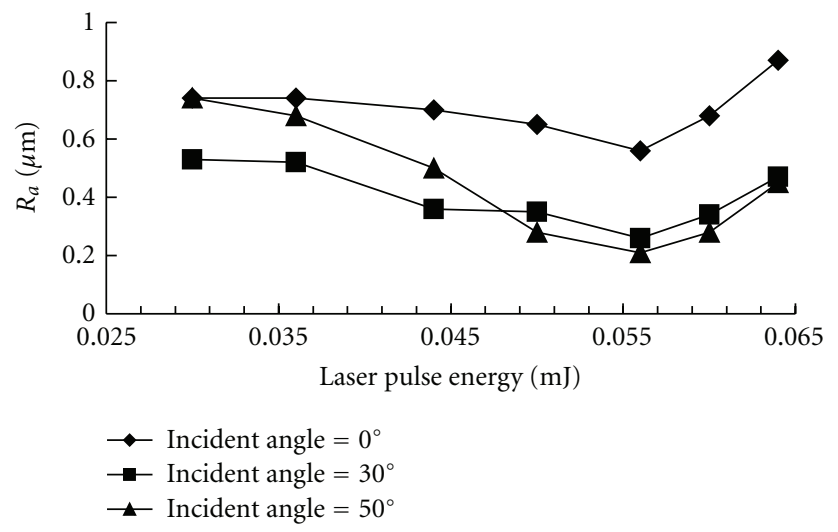

(a) Effect of laser energy on sapphire surface roughness

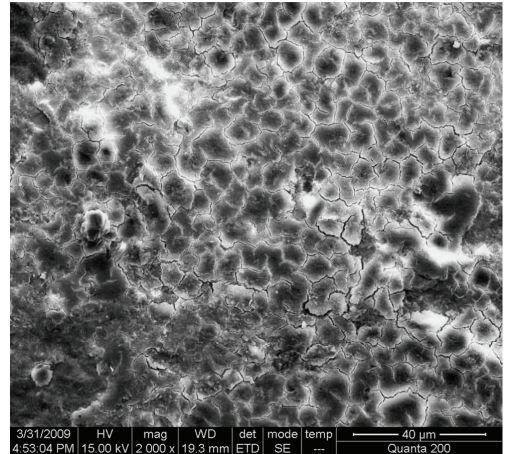

(b) Polished surface $(0.064 \mathrm{~mJ})$

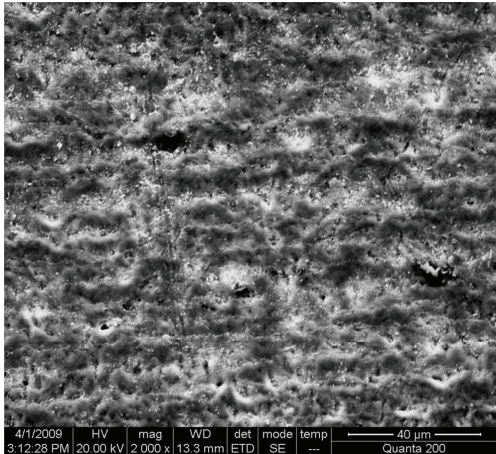

(c) Polished surface $(0.044 \mathrm{~mJ})$

Figure 3: Effect of laser energy on sapphire polishing (pulse repetition rate of $30 \mathrm{kHz}$, scanning speed of $90 \mathrm{~mm} / \mathrm{s}$, single-scan polishing, Sample A, incident angle of $50^{\circ}$ in (b), (c)).

the material is mainly removed by thermal action with lots microcracks, which causes the surface roughness to become worse.

The sapphire material is removed quite a little and the surface cannot be improved obviously if laser energy is too small. With the increase of the pulse energy continuously, the photochemical action by laser radiation increases even accompanied by thermal action of micromelting, and then the surface roughness of polished sapphire decreases prominently. When the pulse energy is higher than the transition point (e.g., $0.056 \mathrm{~mJ}$ in this paper), the thermal action by laser radiation becomes strong and dominant the material removal in the polishing process, which causes lots of microcracks and the surface roughness worse.

\subsection{Effects of Laser Pulse Repetition Rate on Surface Roughness.} The laser pulse repetition rate has obviously influences on pulse energy and then on the surface of sapphire, as shown in Figure 4.

When the pulse repetition rate was of $10 \mathrm{kHz}$, the laser energy became higher and material removal became larger comparing with those with higher pulse repetition rate. As a result, the surface roughness increased, as shown in Figures 4(a) and 4(b). Figure 4(b) also showed some features of deep furrows at the surface, which should be the ablation traces consistent with the laser scanning traces.
When the pulse repetition rate went up to $30 \mathrm{kHz}$, the smoothest surface was obtained. It can be explained that at this condition the laser energy comes to an appropriate value at which the thermal action and photochemical action collaborate favourably. When the pulse repetition rate increased up to $50 \mathrm{kHz}$ or even higher, the laser energy became too small to remove the sapphire material effectively. So the surface roughness of polished sapphire was approximately the same with the original surface, as shown in Figures 4(a) and $4(\mathrm{c})$.

\subsection{Effects on Surface Roughness by Laser Scanning Speed.} The laser scanning speed affects the sapphire polishing by laser-matter interaction time. The faster the laser scanning speed, the shorter the interaction time between the laser and material was and the better the surface roughness became, as shown in Figure 5.

When laser scanning speed as low as $10 \mathrm{~mm} / \mathrm{s}$ was used, the surface material was melted fully because of enough laser-matter interaction time and rapidly resolidified. The material was removed by thermal fracture, resulting in lots of residual microcracks and pits at the surface, as shown in Figure 5(b). So, the surface was very rough (see in Figures 5(a) and 5(b)). The better surface was achieved at the laser scanning speed of $50 \mathrm{~mm} / \mathrm{s}$, as shown in Figure 5(c). When scanning speed went up to $90 \mathrm{~mm} / \mathrm{s}$, the surface 


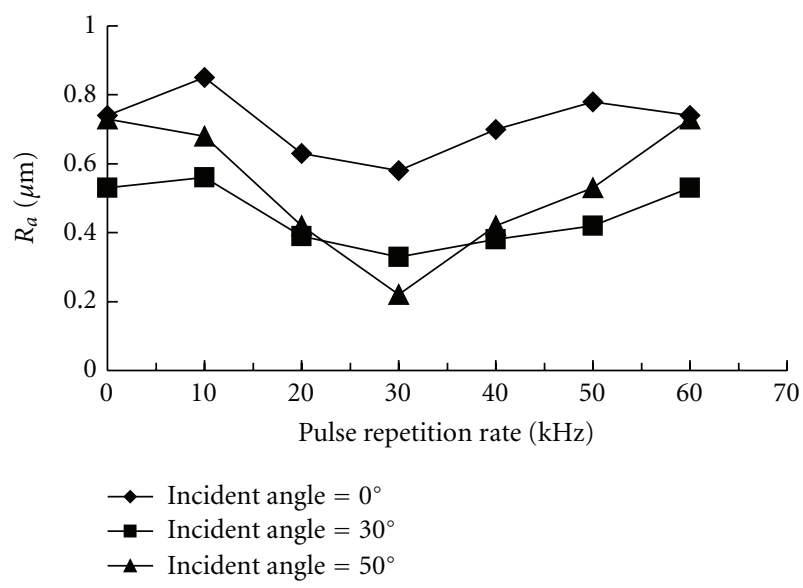

(a) Effect of laser pulse repetition rate on surface roughness

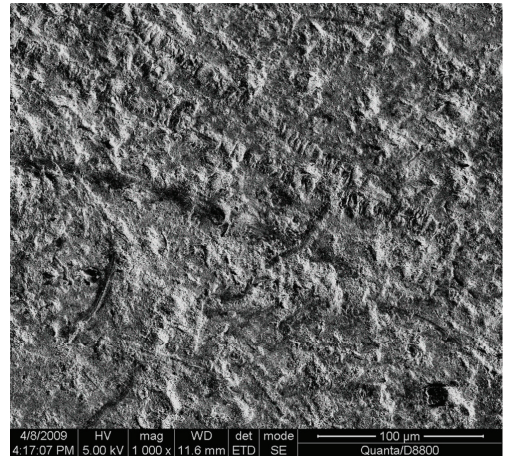

(b) Polished surface $(10 \mathrm{kHz})$

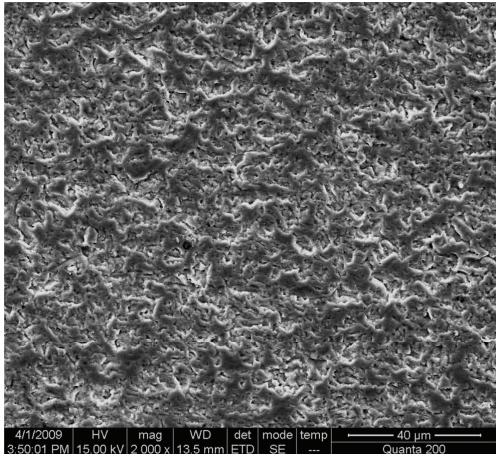

(c) Polished surface $(50 \mathrm{kHz})$

FIGURE 4: Effect of laser pulse repetition rate on sapphire polishing (laser energy of $0.036 \mathrm{~mJ}$, scanning speed of $90 \mathrm{~mm} / \mathrm{s}$, single-scan polishing, Sample A, incident angle of $50^{\circ}$ in (b), (c)).

roughness decreased to the lowest value of $R_{a} 0.27 \mu \mathrm{m}$ and the polished surface was very smooth and dense, as shown in Figure 5(d). When the scanning speed faster than $90 \mathrm{~mm} / \mathrm{s}$ was applied, the surface roughness increased again because the overlapping coefficient of focal spot on the surface became very small.

3.4. Effects of Incident Angle on Surface Roughness. The laser incident angle means the angle between the laser beam and the right direction of the sample surface. Changing incident angle affects the polishing results mainly by changing the focal spot shape of laser light on the radiated surface and then radiated energy density.

With the increase of the laser incident angle, the focal spot shape became elliptic with a little big area and the polished surface trended to became smooth (shown in Figure 6) as the slight decrease of laser energy. Actually, the convex spot of the surface is much easier to be removed under large incident angle than the concave port of the surface, which contributes to the decrease of surface roughness. However, the incident angle greater than 50 degree is difficult to be applied because of experimental set-up limitation in this study.
3.5. Effects of Laser Scanning Patterns on Surface Roughness. Laser scanning pattern has great influence on laser polishing of sapphire. Variation of scanning patterns will affect the overlapping ratio of focal spot. Five types of scanning patterns were considered in this study.

Pattern A: single-laser beam scans along one direction, as shown in Figure 7(a).

Pattern B: laser beam scans along one direction firstly, and then along the same direction after the sample rotates by $\theta$ of $90^{\circ}$, as shown in Figure 7(b).

Pattern C: laser beam scans along one direction firstly, and then along the same direction after the sample rotates by $\theta$ of $75^{\circ}$.

Pattern D: laser beam scans along one direction for twice, as shown in Figure 7(a).

Pattern E: laser beam scans firstly along one direction for twice, and then along the same direction for twice after the sample rotates by of $90^{\circ}$, as shown in Figure 7(b).

Figure 8 shows the effects of different laser beam scanning patterns on sapphire surface roughness. Surface 


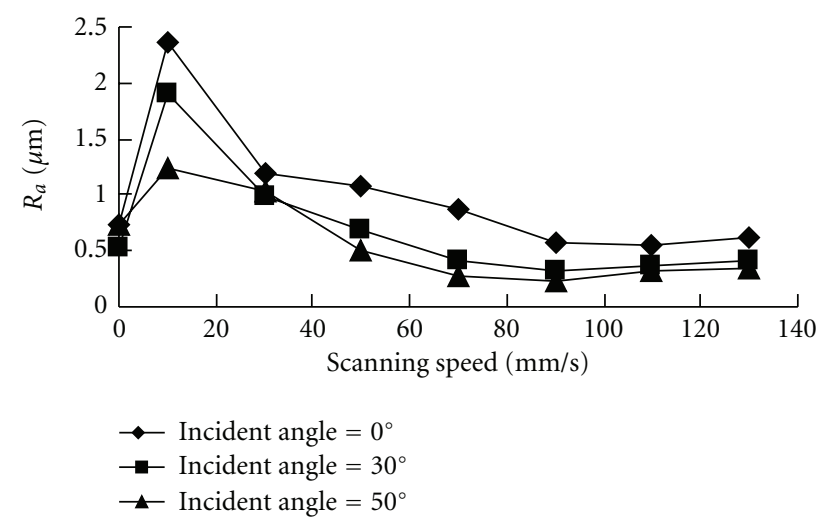

(a) Effect of laser scanning speed on sapphire surface roughness

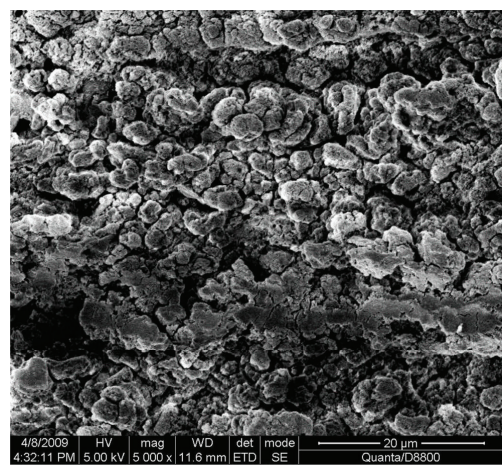

(b) Polished surface $(10 \mathrm{~mm} / \mathrm{s})$

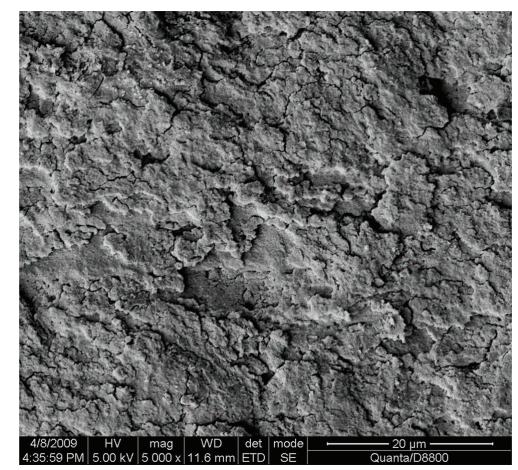

(c) Polished surface $(50 \mathrm{~mm} / \mathrm{s})$

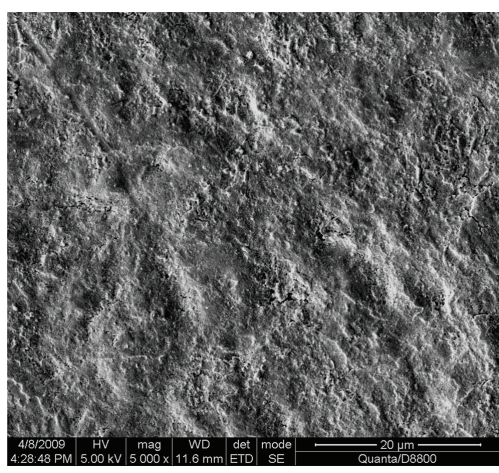

(d) Polished surface $(90 \mathrm{~mm} / \mathrm{s})$

FIGURE 5: Effect of scanning speed on sapphire polishing (laser energy of $0.036 \mathrm{~mJ}$, pulse repetition rate of $30 \mathrm{kHz}$, single-scan polishing, Sample A, incident angle of $50^{\circ}$ in (b), (c), and (d)).

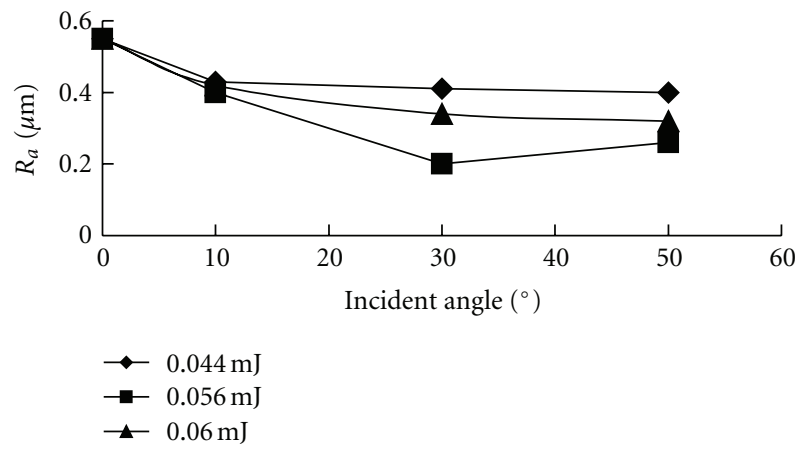

Figure 6: Effect of incident angle on sapphire surface roughness (pulse repetition rate of $30 \mathrm{kHz}$, scanning speed of $90 \mathrm{~mm} / \mathrm{s}$, single-scan polishing, Sample A).

roughness after polishing with scanning pattern A was lower than those polished by the other four scanning patterns. This is because single scanning can remove the convex spot sufficiently. Multiple scanning may remove both the convex and concave spots and produces deep furrow, which results in the increasing of surface roughness, as shown in Figures $8(\mathrm{~b})$ and $8(\mathrm{c})$. Surface roughness after polishing with scanning pattern $\mathrm{B}$ was lower than that of scanning patterns C. This means that the smaller intersection angle, the lower surface roughness can obtained.
3.6. Effects of Initial Surface Conditions on Surface Roughness. Three samples with different surface conditions were prepared for this research, namely, mechanical lapped, sawed, and green laser $(532 \mathrm{~nm})$ polished surface, as shown in Figure 2.

After being polished by ultraviolet laser, the surface roughness of the mechanical lapped sample dropped by 50 percent, which is 36 percent higher than that of green laser polished sample and 20 percent higher than that of sawed sample. Figure 9 shows the effects of initial sapphire 


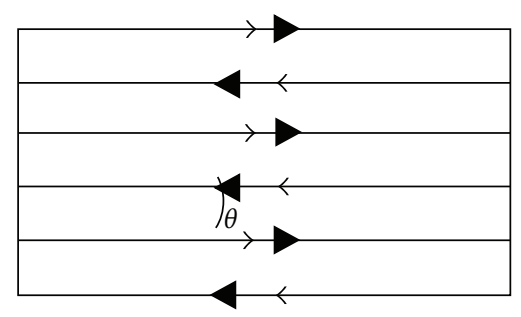

$\rightarrow$ First scanning direction

$\rightarrow$ Second scanning direction

(a) Parallel scanning

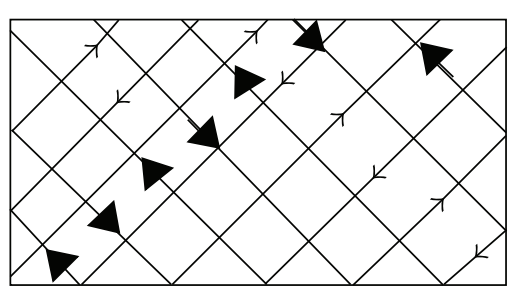

$\rightarrow$ First scanning direction

$\rightarrow$ Second scanning direction

(b) Intersectional scanning

FIGURE 7: Laser beam scanning patterns.

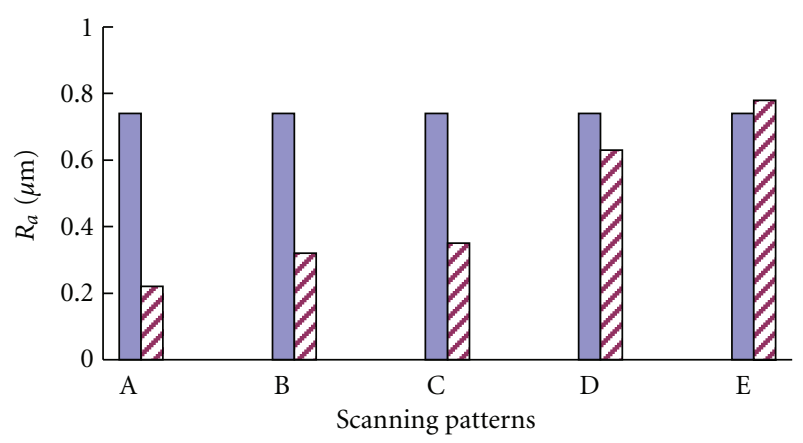

$\square$ Before polishing

$\square$ After polishing

(a) Effects of scanning patterns on sapphire surface roughness

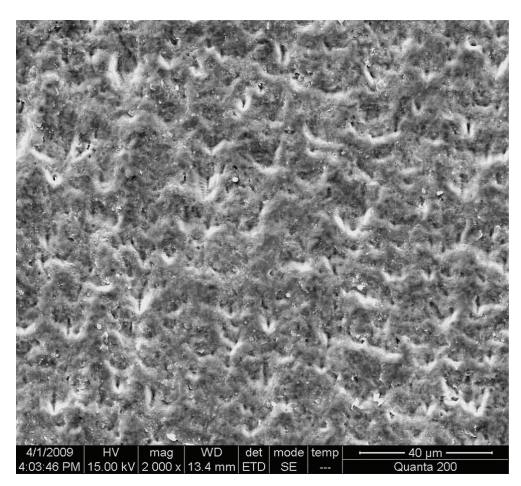

(b) Polished with Pattern C

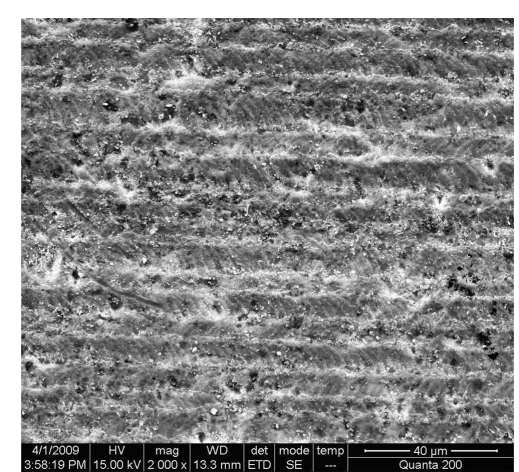

(c) polished with Pattern D

FIGURE 8: Effect of laser scanning patterns on sapphire polishing (laser energy of $0.056 \mathrm{~mJ}$, scanning speed of $90 \mathrm{~mm} / \mathrm{s}$, pulse repetition rate of $30 \mathrm{kHz}$, incident angle of $50^{\circ}$, Sample A).

surface conditions on the surface roughness. The smaller roughness the initial surface of sample, the larger the reduced rate of surface roughness was obtained after ultraviolet laser polishing. It is indicated that ultraviolet laser polishing is more appropriate for precision polishing.

\section{Conclusion}

(1) With the increase of laser energy, the surface roughness $R_{a}$ decreases when laser energy is lower than $0.056 \mathrm{~mJ}$ and increases again when the energy is higher than $0.056 \mathrm{~mJ}$ because of different laser actions.

(2) The faster the laser scanning speed, the shorter the interaction time is. The surface becomes rougher when scanning speed is as low as 10 and $30 \mathrm{~mm} / \mathrm{s}$.

(3) With the increase of the laser incident angle, the surface roughnes tends to decrease slightly.

(4) Single-scanning pattern is superior to multiplescanning pattern and intersectional scanning pattern. 


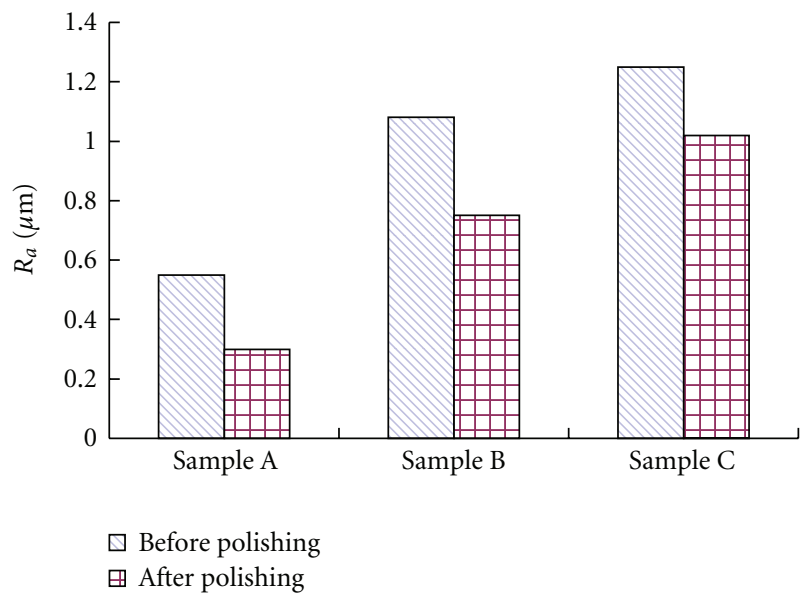

FIGURE 9: Effects of different original sapphire surface conditions on surface roughness.

The smaller intersection angle, the lowersurface roughness obtained after laser polishing.

(5) The smaller roughness of the initial surface of sample, the larger percentage of roughness decreases after ultraviolet laser polishing. It is indicated that ultraviolet laser polishing is more suitable for precision polishing.

(6) Sapphire surface roughness $R_{a}$ can be decreased from 0.74 to $0.2 \mu \mathrm{m}$ by ultraviolet laser polishing under the condition of pulse width of $20 \mathrm{~ns}$, pulse energy of $0.056 \mathrm{~mJ}$, pulse repetition rate of $30 \mathrm{kHz}$, scanning speed of $90 \mathrm{~mm} / \mathrm{s}$, incident angle of 50 degree and patterns A for mechanical lapped surface in this experiment.

\section{Acknowledgments}

The research was supported by Nature Science Foundation of China (nos. 50675038, 50805027), Science and Technology Plan Project of Guangdong Province (no. 2004B10201018), and Science and Technology Plan Project of Guangzhou (no. 2005J1-C0081).

\section{References}

[1] H. Park and H. M. Chan, "A novel process for the generation of pristine sapphire surfaces," Thin Solid Films, vol. 422, no. 1-2, pp. 135-140, 2002.

[2] X. Guo, X. Wei, and X. Xie, "Development of sapphire polishing technology," Mechanical \& Electrical Engineering Technology, vol. 35, no. 9, pp. 76-78, 2006.

[3] Z. Li, P. Li, J. Fan, R. Fang, and D. Zhang, "Energy accumulation effect and parameter optimization for fabricating of highuniform and large-area period surface structures induced by femtosecond pulsed laser," Optics and Lasers in Engineering, vol. 48, no. 1, pp. 64-68, 2010.

[4] I.-B. Sohn, Y.-C. Noh, S.-C. Choi, D.-K. Ko, J. Lee, and Y.-J. Choi, "Femtosecond laser ablation of polypropylene for breathable film," Applied Surface Science, vol. 254, no. 16, pp. 4919 4924, 2008.
[5] M. Folwaczny, A. Mehl, C. Haffner, and R. Hickel, "Polishing and coating of dental ceramic materials with $308 \mathrm{~nm}$ XeCL excimer laser radiation," Dental Materials, vol. 14, no. 3, pp. 186-193, 1998.

[6] M. Udrea, H. Orun, and A. Alacakir, "Laser polishing of optical fiber end surface,” Optical Engineering, vol. 40, no. 9, pp. 20262030, 2001.

[7] T. M. Shao, M. Hua, H. Y. Tam, and E. H. M. Cheung, "An approach to modelling of laser polishing of metals," Surface and Coatings Technology, vol. 197, no. 1, pp. 77-84, 2005.

[8] X. Wei, X. Guo, and X. Xie, "Influence of the condition parameters on UV pulsed laser polishing of sapphire wafer," in Proceedings of the 8th Pacific Rim Conference on Lasers and ElectroOptics (CLEO/Pacific Rim '09), September 2009. 

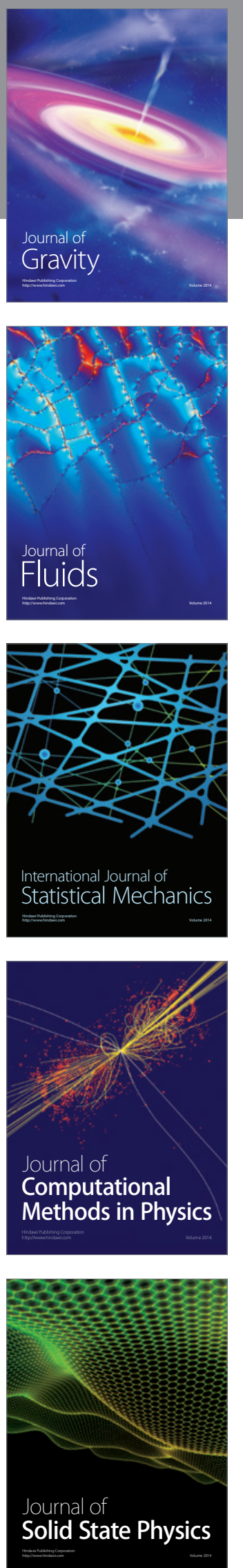

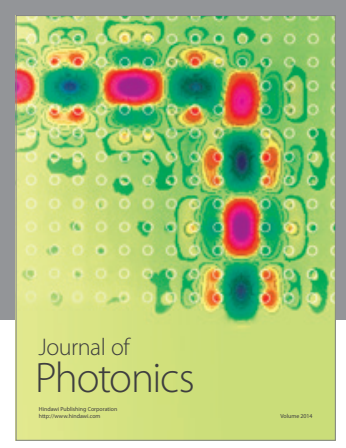

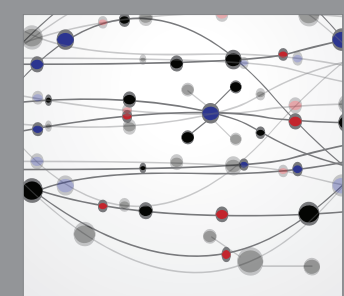

The Scientific World Journal
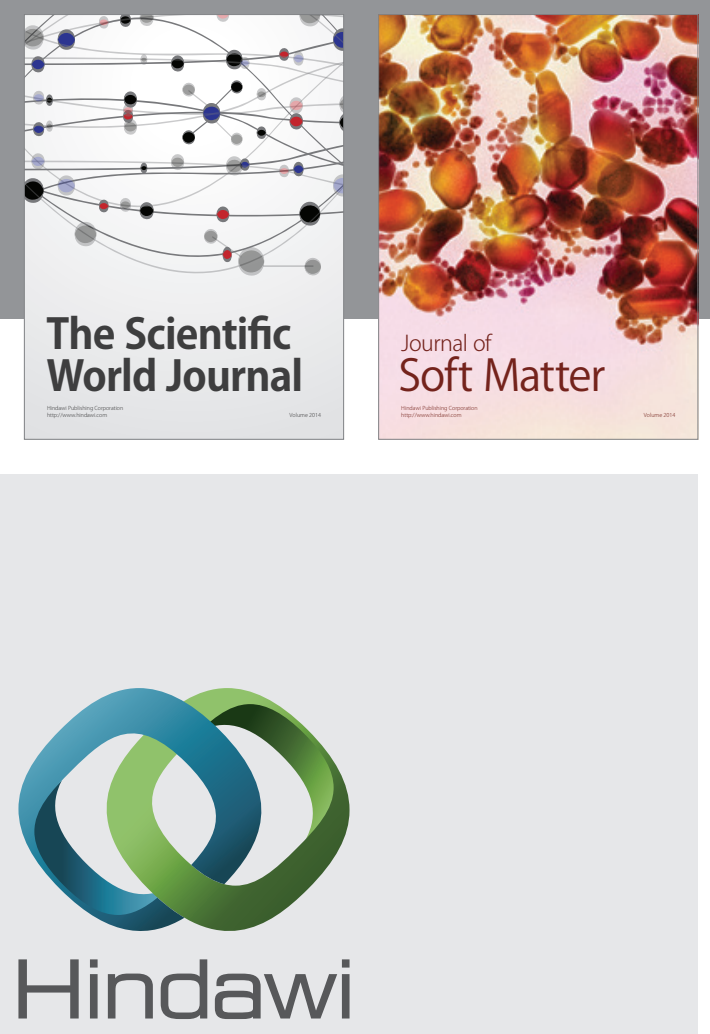

Submit your manuscripts at

http://www.hindawi.com
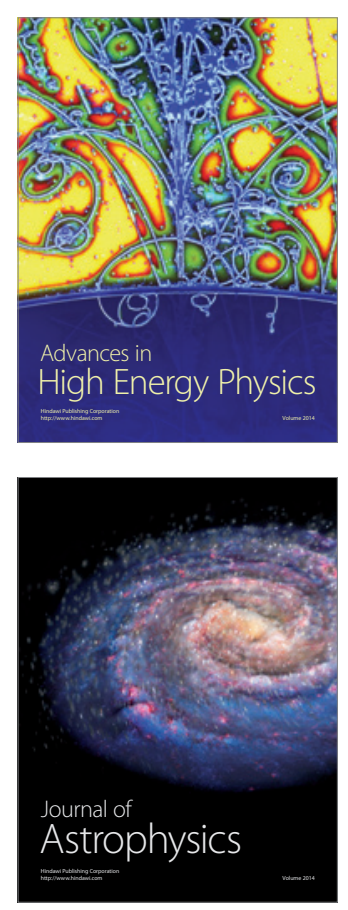
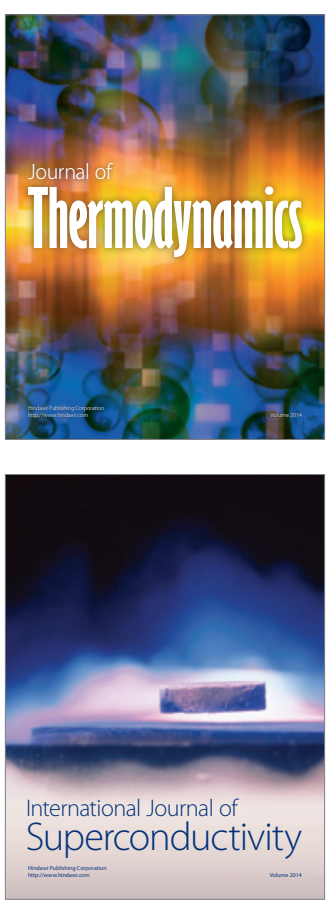
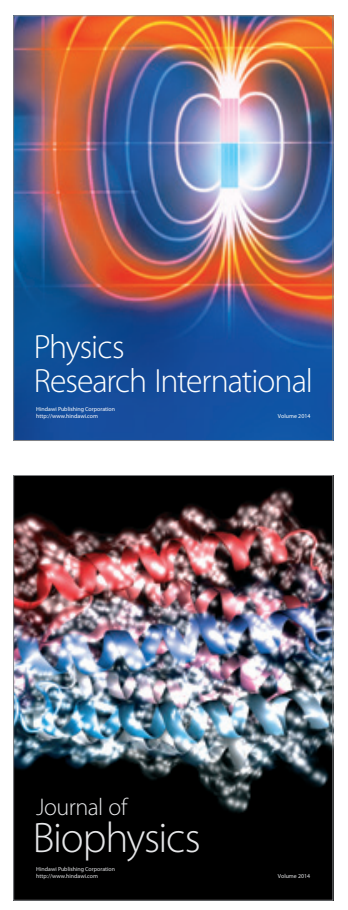
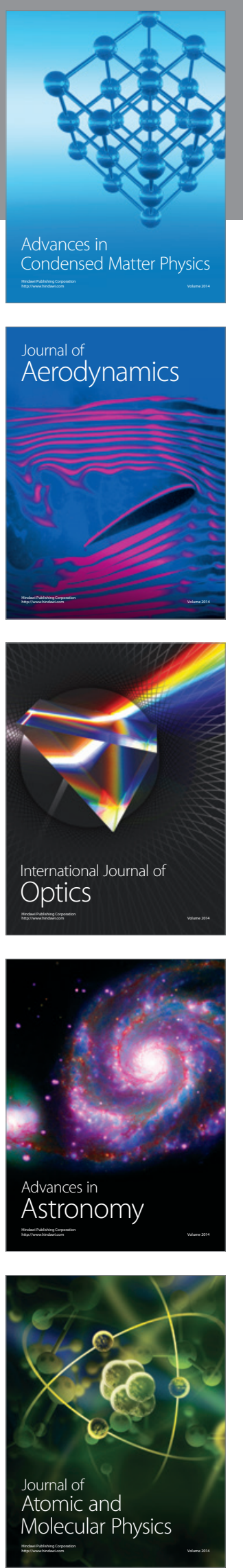